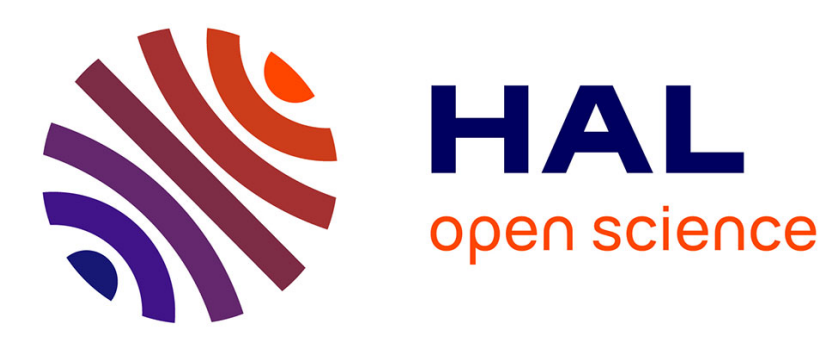

\title{
Remote online testing of embedded systems using Optical BILBO
}

\author{
Abdelhakim Latoui, Raphael Canals
}

\section{To cite this version:}

Abdelhakim Latoui, Raphael Canals. Remote online testing of embedded systems using Optical BILBO. Electronic Conference (BEC), 2014 14th Biennial Baltic, Oct 2014, Tallinn, Estonia. pp.101104, 10.1109/BEC.2014.7320566 . hal-01229930

\section{HAL Id: hal-01229930 https://hal.science/hal-01229930}

Submitted on 1 Dec 2015

HAL is a multi-disciplinary open access archive for the deposit and dissemination of scientific research documents, whether they are published or not. The documents may come from teaching and research institutions in France or abroad, or from public or private research centers.
L'archive ouverte pluridisciplinaire HAL, est destinée au dépôt et à la diffusion de documents scientifiques de niveau recherche, publiés ou non, émanant des établissements d'enseignement et de recherche français ou étrangers, des laboratoires publics ou privés. 


\title{
Remote Online Testing Of Embedded Systems Using Optical BILBO
}

\author{
Abdelhakim LATOUI ${ }^{1,2} \&$ Raphael CANALS ${ }^{2}$ \\ ${ }^{1}$ Department of Electronics, Faculty of Science \& Technology \\ University of Mohamed El Bachir El Ibrahimi, Bordj Bou Arréridj, Algeria, 34030. \\ ${ }^{2}$ PRISME, University of Orléans, 12 rue de Blois- F- 45067 Orleans Cedex 2. \\ E-mail:latoui.ahakim@yahoo.fr,raphael.canals@univ-orleans.fr
}

\begin{abstract}
In this paper, an online testing approach that checks continuously at remote system the correctness of embedded systems in full operation is proposed. Our new method exploits optical beams produced by an Optical Built-In Logic-Block Observation (OBILBO) register based on the captured data presented on the outputs of all stages of the registers in the BILBO. Then it is possible to send the captured data to remote system equipped with optical sensors in real time way. The final captured response data can also be used for final comparison with stored data of a golden circuit. Preliminary simulation results showed that faults are concurrently detected without affecting normal system operation and without having recourse to any external or internal Automatic Test Pattern Generation (ATPG).
\end{abstract}

Keywords-Embedded Systems, On-Line Testing, Test and Testability, VHDL-AMS, Optical Sensors.

\section{INTRODUCTION}

Nowadays, embedded systems are omnipresent in many applications whose contributions are significant in terms of weight, size, power consumption, response time and flexibility. Although their sophistication continues to increase, embedded systems are increasingly used in critical applications (e.g. medical or transport applications) in which their misbehavior may generate unacceptable consequences that may go up to human life loss [1]. It is therefore vital to ensure immediate detection of errors caused by a failure in these systems by having recourse to online testing. These tests can check continuously the results produced by these systems in exploitation mode to confirm the validity of their functioning [2]. However, this kind of online test constitutes nowadays a big challenge for test designers of such systems.

So, a large variety of on-line testing techniques has been proposed in the literature $[3,4]$. Such techniques are for instance: self-checking design, signature monitoring, on-line monitoring of reliability relevant parameters such as current or temperature, implementation of BIST techniques specific to on line testing, etc.

However, in modern System-on-Chip (SoC) design, many cores are integrated into a single chip. Some of them are embedded and cannot be accessed directly from the outside of the chip. Such SoC designs make the test of these embedded cores a great challenge. Although the BIST technique is one of the most popular solution $[5,6]$ adopted for the test of embedded cores, it is used to realize a discontinuous online test by activating periodically the necessary test sequences. Detection of the faults is then carried out with a delay which is related to the periodicity of the test.

Furthermore, the International Technology Roadmap for Semiconductors (ITRS) [7] projects that a Giga-Scale Integration (GSI) chip manufactured at the $18 \mathrm{~nm}$ technology node will have a chip-to-board speed of $56 \mathrm{GHz}$. To this end, it identifies optical interconnects as a potential enabling technology. Recent reports relating to the monolithic fabrication of photo detectors, modulators and emitters on Si have given rise to the exciting prospect of combining optoelectronics and GSI in a high-volume manufacturing environment [8]. In fact, the domain which involves measuring the electrical quantity such as voltage or current, using optical methods is adopted to perform testing modern integrated circuits [9]. The technology that uses electro-optic (EO) probing of electrical circuits with laser beam is developed by several research teams [10]. These techniques have for object the characterization of off-line circuits. EO probing can also be used, as detailed in [11,12], to make real time measurement of the state (i.e. high or low) of digital signals present at the outputs or the inputs of a circuit board or integrated chip in order to detect any failure of the latter. So, the combination of BIST approach and EO probing can lead to viable solution for on-line testing of nowadays circuits. For example, in [13], an Optical Built-In Logic-Block Observation (OBILBO) was proposed to achieve on line detection of stuck at fault of embedded systems with very low error latency. Nevertheless, the solution has an area overhead due to the use of the hardware duplication.

This paper presents a more efficient solution in terms of area overhead. The novel proposed approach is based on the OBILBO which permits the emission at remote system of optical beams according to the logical states of its outputs. When operating in parallel load mode, the OBILBO acts as snapshot of outputs (inputs) data of the Circuit Under Test (CUT). Thus, the transmitted data, corresponding to the outputs (inputs) of the CUT, via the OBILBO, can be compared at remote system in a real time way to the stored data of a Reference Model (RM) of the CUT. The stored data replace and thus eliminate the complete duplication of the CUT.

The rest of the paper is organized as follows. Section 2 describes the new remote on-line testing scheme. The corresponding VHDL-AMS model is presented in Section 3. Analysis and experimental results are presented in Section 4. Section 5 concludes this paper.

\section{Remote Online Testing Scheme}

An ideal on-line testing scheme would have $100 \%$ error coverage, error latency of 1 clock cycle, no space redundancy, and no time redundancy. Considering all four parameters in the design of an on-line testing scheme may create conflicting goals. High coverage, for example, requires high error latency, space redundancy and/or time redundancy. On the other hand, schemes with immediate detection (error latency equaling 1) minimize time redundancy but require more hardware [14]. However, reducing the error latency is often considered as a primary goal in on-line testing [15]. As a matter of fact, techniques using COTS (Component-Off-The-Shelfsuch) as duplication or triplication are 


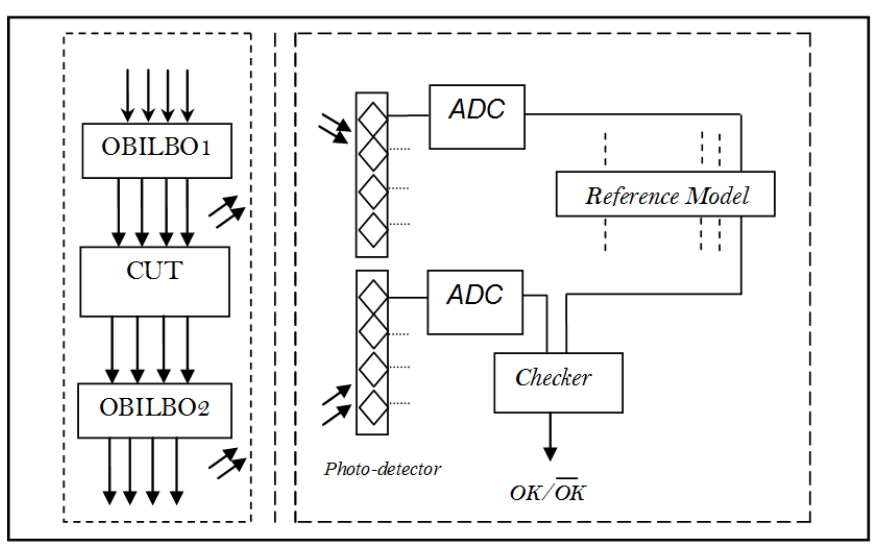

Figure 1. Schematic representation of the proposed method.

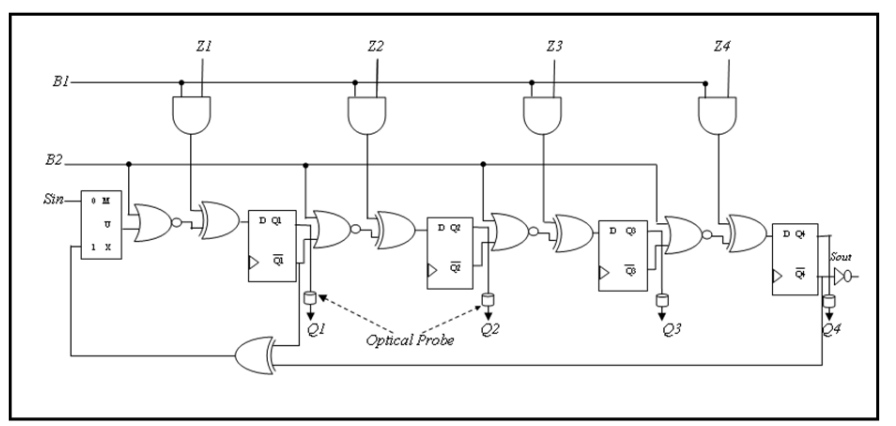

Figure 2. Logic diagram of OBILBO.

more often adopted even at the extra area overhead, since they have low error detection latency compared to others techniques described earlier [3]. This paper presents a new on line testing scheme that allows errors detection with very low error latency and minimum area overhead.

The overall scheme of the proposed approach is illustrated in Figure 1 where the CUT can be any chip supporting BuiltIn Logic-Block Observation (BILBO) architecture. The BILBO is an embedded and off-line BIST architecture because it uses existing flip-flops from the CUT to construct the TPG (Test Pattern Generator) and ORA (Output Response Analyzer) functions [16]. Optical probes are then added to the BILBO register to form an OBILBO [13]. In addition to the standards functions [16] of the BILBO, the OBILBO continuously produces optical beams according to the logic states of its outputs thanks to optical probes integrated in its architecture. Figure 2 [13] shows the logic diagram of four stages OBILBO register.

To ensure real time checking of the CUT integrity without affecting its normal operation, the OBILBO1 and OBILBO2 (Figure 1) should be however configured in their parallel load mode. Thus, OBILBO1 and OBILBO2 produce optical signals corresponding to the logical states of the CUT inputs and outputs respectively. The transmitted optical signals can then be captured at remote system equipped with photo-detectors, such as PIN diodes, and Analog to Digital Converters (ADC). The final captured data are compared to the stored data of a RM, i.e. for each input data vector (corresponding to signals transmitted by OBILBO1), we establish a corresponding reference response data vector which is compared to the response data (corresponding

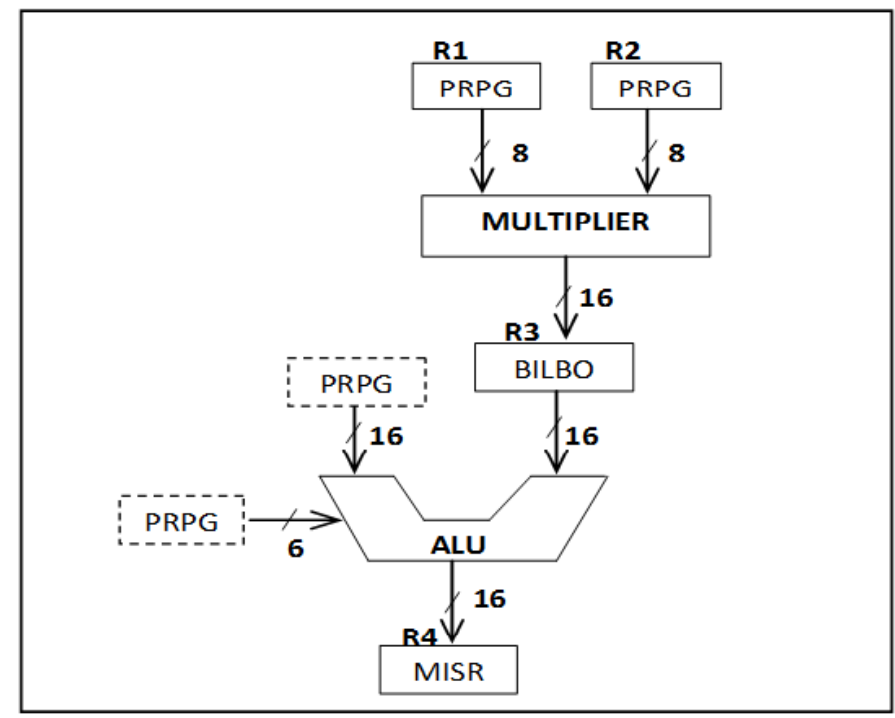

Figure 3. Portion of the TMS32010 Signal-processing chip.

to the signals transmitted by OBILBO2) of the CUT. It is worth noting that the constituting elements of the proposed scheme exchange signals which are of nature heterogeneous. In the next section, we give a possible modeling of the described scheme with the VHDL-AMS language.

\section{VHDL-AMS MODELING}

The VHDL-AMS language is an extension of the IEEE 1076 (VHDL) standard for describing digital, analog and mixedsignal systems. It provides mixed-disciplines modeling, wherein different domains can be described and simulated in a single entity. In fact, the system of the Figure 1 can be viewed as simple components connections which can be modeled using components instantiation.

Signal-processing chips are basic elements in today Embedded and Real-Time systems. A typical example of these is the TMS32010 signal-processing chip which supports BILBO architecture. Indeed, various BIST architectures were applied to this chip in order to achieve offline testing. Four BIST designs are proposed in [5]. Among these architectures a BILBO solution was proposed as shown in Figure 3 in which some registers are replaced by BILBO registers to test the multiplier and the ALU. In our case, all MISR and PRPG are considered as BILBO registers which are all replaced now by OBILBO registers. However, in order to test this chip in on-line mode, the CUT of Figure 1 is considered now as portion of the TMS32010 signalprocessing chip with its novel OBILBO registers, as depicted in Figure 4.

We assume now that the ALU of TMS32010 is considered as single adder cell as illustrated in Figure 4 (since more signals are required to represent the simulation results of the ALU). We suppose that the adder is faulty due the stuck- at- 0 at the input of the grey OR-gate. Table 1 shows the results which should be produces in the faulty free case and in the faulty one. The corresponding VHDL-AMS model is given in Figure 5. 


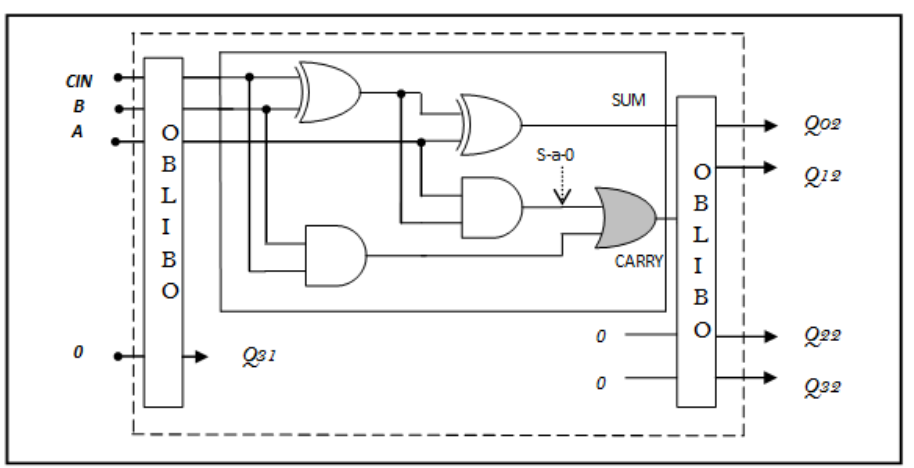

Figure 4. Full Adder with OBILBO.

Table I. TRUTh TABLE

\begin{tabular}{|c|c|c|c|c|c|c|}
\hline$A$ & $B$ & CIN & SUM (Free) & SUM (Faulty) & Carry (Free) & Carry (Faulty) \\
\hline \hline 0 & 0 & 0 & 0 & 0 & 0 & 0 \\
\hline 0 & 0 & 1 & 1 & 1 & 0 & 0 \\
\hline 0 & 1 & 0 & 1 & 1 & 0 & 0 \\
\hline 0 & 1 & 1 & 0 & 0 & 1 & 0 \\
\hline 1 & 0 & 0 & 1 & 1 & 0 & 0 \\
\hline 1 & 0 & 1 & 0 & 0 & 1 & 0 \\
\hline 1 & 1 & 0 & 0 & 0 & 1 & 1 \\
\hline 1 & 1 & 1 & 1 & 1 & 1 & 1 \\
\hline
\end{tabular}

\section{ANALysis And EXPERIMENTAL RESUlts}

Simulation results are reported in Figure 6 where $b 1[2]$ and $b 1[1]$ are the control lines of OBILBO1 whereas $a, b$ and cin are its inputs data. $q 1[2], \ldots q 1[0]$ are the outputs of OBILBO1 where their corresponding optical signals are $q l 21, \ldots q l 01$ respectively which are also the inputs of the first set of the PIN diodes.

The ADCs placed after this first set deliver the digital signals $d 21, d 11$ and $d 01$ which correspond in fact to the inputs data $a, b$ and cin respectively. However, $d 02$ and $d 12$ are the signals delivered by the second set of ADC placed after the second set of the PIN diodes which correspond to the sum and the carry produced by the faulty adder respectively. The optical signals for the second set are not shown in the figure. The signals $s 1$ and $c 1$ are the sum and the carry references delivered according to the inputs data $d 01, d 11$ and $d 12$ which are in fact the converted data of the optical beams produced by OBILBO1 (corresponding to the inputs data $a, b$ and $\operatorname{cin}$ ).

The checker has delivered the signals $\operatorname{er} 0$ and $\operatorname{er} 1$ where $\operatorname{er} 0$ is the result of comparison between the $s 1$ (reference sum) and $d 02$ (corresponding to the faulty sum) and er 1 is the result of comparison between $c 1$ and $d 12$ (corresponding to the faulty carry). We note that er 0 is equal to 0 when $s 1$ and $d 02$ are identical and equal to 1 otherwise (Idem for er 1 ). We can see from these results that the considered stuck-at- 0 is detected after only one clock cycle (delay introduced by the OBILBO) with no having recourse to any ATPG. However, for a duplication technique errors are not detected until they propagate to the CUT outputs. For the sake of argument, considering the system of Figure 3, if we assume that the inputs of R1 and R2 are the inputs of the CUT and the outputs of $R 4$ are its outputs, a fault at the input of the multiplier cannot be detected until it propagates to the outputs of $R 4$. Therefore, the ALU and the $R 4$ register will introduce a delay like for the multiplier and the $R 3$ register. Thus, more clock cycles are required to detect this fault. As a result, the error latency will increase and it will
LIBRARY IEEE, DISCIPLINES;

USE DISCIPLINES.ELECTROMAGNETIC_SYSTEM.ALL;

USE IEEE.MATH_REAL.ALI;

ENTITY BENCH_ALU IS

END BENCH_ALU;

ARCHITECTURE ARCH OF BENCH ALU IS

SIGNAL CLK, RESET1, SCAN IN1, SCAN OUT1:BIT;

SIGNAL RESET2, SCAN_IN2, SCAN_OUT $\overline{2}: B I T$;

SIGNAL B1,B2 : BIT_VECTOR-̄2 DOWNTO 1);

SIGNAL Z1, Z2, Q1, Q2: BIT VECTOR ( 3 DOWNTO 0):

TERMINAL N01, N11, N21, N31, N02 , N12, N22, N32:

ELECTRICAL;

SIGNAL S01,S11,S21,S31,D01,D11,D21,D31： BIT;

SIGNAL $\mathrm{S} 02, \mathrm{~S} 12, \mathrm{~S} 22, \mathrm{~S} 32, \mathrm{D} 02, \mathrm{D} 12, \mathrm{D} 22, \mathrm{D} 32: \mathrm{BIT}$;

SIGNAL A, B, CIN, S1, C1, S2, C2, G0,G1, G2, G3: BIT;

SIGNAL SIG,SIG01, SIG11,SIG21,ER0,ER1,ER2,ER3: BIT;

QUANTITY QL01, QL11, QL21, QL31: REAL;

QUANTITY QL02, QL12, QL22, QL32: REAL ;

BEGIN

$\mathrm{S} 01<=01(0) ; \mathrm{S} 11<=01(1) ; \mathrm{S} 21<=01(2) ; \mathrm{S} 31<=01(3)$;

$\mathrm{S} 02<=\mathrm{Q} 2(0) ; \mathrm{S} 12<=\mathrm{Q} 2(1) ; \mathrm{S} 22<=\mathrm{Q} 2(2) ; \mathrm{S} 32<=\mathrm{Q} 2$ (3) ;

$\mathrm{Z} 1(0)<=\mathrm{CIN} ; \mathrm{Z} 1(1)<=\mathrm{B} ; \mathrm{Z} 1(2)<=\mathrm{A} ; \mathrm{Z} 1(3)<=$ ' $^{\prime}$ ' ;

$\mathrm{Z} 2(0)<=\mathrm{S} 2 ; \mathrm{Z} 2(1)<=\mathrm{C} 2 ; \mathrm{Z} 2(2)<={ }^{\prime} 0^{\prime} ; \mathrm{Z} 2(3)<=0^{\prime}$;

--OBILBO 1

BILB1:ENTITY BILBO (ARCH) PORT MAP

(RESET1, CLK, SCAN_IN1, SCAN_OUT1, B1, Z1, Q1);

OPP01:ENTITY OP_PROBE PORT MAP (S01, QL01);

PIND01:ENTITY PIN_DIODE PORT MAP

(N01, ELECTRICAL GROUND, QL01);ADC01: ENTITY ADC PORT

MAP (N01, ELECTRICAL_GROUND, D01);

OPP11:ENTITY OP_PROBE PORT MAP (S11, QL11);

PIND11:ENTITY PIN DIODE PORT MAP

(N11, ELECTRICAL_GE'TOUN, QL11);ADC11: ENTITY ADC PORT

MAP (N11, ELECTRICAL_GROUND, D11);

OPP21:ENTITY OP PROBE PORT MAP (S21, QL21);

PIND21:ENTITY PIN_DIODE PORT MAP

(N21, ELECTRICAL_GROUND, QL21); ADC21:ENTITY ADC PORT MAP (N21, ELECTRICAL_GROUND , D21);

OPP31:ENTITY OP PROBE PORT MAP (S31, QL31);

PIND31:ENTITY $\bar{P} I N$ DIODE PORT MAP

(N31, ELECTRICAL_GROUND, QL31);ADC31: ENTITY ADC PORT MAP (N31, ELECTRICAL_GROUND , D31) ;

-OBILBO 2

BILB2: ENTITY BILBO (ARCH) PORT MAP

(RESET2, CLK, SCAN_IN2,SCAN_OUT2, B2, Z2, Q2);

OPP02:ENTITY OP_PROBE PORT MAP (S02, QL02);

PIND02:ENTITY PIN DIODE PORT MAP

(N02, ELECTRICAL GROUND, QL02); ADC02: ENTITY ADC PORT

MAP (N02, ELECTRICAL_GROUND , D02);

OPP12:ENTITY OP_PROBE PORT MAP (S12,QL12);

PIND12:ENTITY PIN DIODE PORT MAP

(N12, ELECTRICAL_GROUND, QL12);ADC12: ENTITY ADC PORT

MAP (N12,ELECTRICAL_GROUND, D12);

OPP2 2: ENTITY OP PROBE PORT MAP (S22, QL22);

PIND22:ENTITY PIN_DIODE PORT MAP

(N22, ELECTRICAL_GROUND, QL22);ADC22: ENTITY ADC PORT

MAP (N22, ELECTRICAL GROUND , D22) ;

OPP32:ENTITY OP PROBE PORT MAP (S32, QL32);

PIND32:ENTITY PIN_DIODE PORT MAP

(N32, ELECTRICAL GROUND, QL32); ADC32:ENTITY ADC PORT

MAP (N32, ELECTRICAL GROUND , D32);

GOLDO: ENTITY CHECKERT PORT MAP (CLK, D01, GO);

GOLD1: ENTITY CHECKERT PORT MAP (CLK, D11,G1);

GOLD2: ENTITY CHECKERT PORT MAP (CLK, D21,G2):

GOLD3: ENTITY CHECKERT PORT MAP (CLK, D21,G3);

AD_G:ENTITY ADD_GOLD PORT MAP (G0,G1,G2,S1,C1);

AD F:ENTITY ADD_F PORT MAP

(SIG01, SIG11, SIG21, S2, C2) ;

$\mathrm{SIG} 01<=\mathrm{Q} 1(0) ; \operatorname{SIG} 11<=\mathrm{Q} 1(1) ; \mathrm{SIG} 21<=\mathrm{Q} 1(2) ; \mathrm{SIG}<=$ ' ' $^{\text {; }}$

CHECK0:ENTITY CHECKER PORT MAP(CLK,D02,S1,ERO);

CHECK1:ENTITY CHECKER PORT MAP (CLK, D12,C1,ER1);

CHECK2: ENTITY CHECKER PORT MAP (CLK, D22, G2,ER2);

CHECK 3 : ENTITY CHECKER PORT MAP (CLK, D22, G3, ER3);

END ARCH;

Figure 5. VHDL-AMS Model of the proposed scheme. 
increase more and more with the complexity of the CUT. It follows that it is clear the proposed solution is more efficient since it reduces considerably the error detection latency with minimum area overhead and also permits a comparison at remote system, contrary to the duplication technique.

\section{CONCLUSION}

In this work, a new remote online testing scheme for embedded systems based on the Optical BILBO (OBILBO) is proposed. Simulation results show that the proposed solution not only ensures errors detection concurrently to the normal function of the CUT with very low error latency but also requires less area overhead, compared to the duplication technique. Then, the optical technology is promising for online testing of embedded systems but it may be nevertheless accompanied by other problems due mainly to interferences.

\section{REFERENCES}

[1] Yue Ma; Wei Jiang; Nan Sang; Ziguo Zhong, "An Adaptive Risk Control and Security Management for Embedded Real-Time System," In 2012 Seventh International Conference, Availability, Reliability and Security (ARES), pp. 11-17, 2012.

[2] Ferranti, E., Steiger, R., Jain, R.; Nagy, K., "CAST: Automating Software Tests for Embedded Systems," In 2012 IEEE Fifth International Conference, Software Testing, Verification and Validation (ICST), pp. 457-466, 2012.

[3] Nicolaids.M and Zorian "On-Line Testing for VLSI- A Compendium of Approaches “, Journal of Electronic Testing Theory and Application, no. 12, pp.7-20, 1998.

[4] Papa, G., Garbolino, A.-T.T., " Optimal On-Line Built-In Self-Test Structure for System-Reliability Improvement", IEEE Congress on Evolutionary Computation (CEC), pp. 222-229, 2011.

[5] Abramovici. M, M.A. Breuer, A.D. Friedman, "Digital Systems Testing and Testable Design", IEEE Press, 1990.

[6] S. Mourad and Y. Zorian, "Principles of Testing Electronic Systems", John Wiley \& Sons, Somerset, NJ, 2000.

[7] Semiconductor Industry Association, "The International Technology Roadmap for Semiconductors," 2003.

[8] Thacker, H.D., Ogunsola, O.O., Muler, A.V., Meindl, J.D., "Wafer-Testing of Optoelectonic-Gigascale CMOS Integrated Circuits", IEEE Journal of Selected Topics in Quantum Electronics, Vo. 17, no. 3, pp. 659-670, 2011.

[9] J.C. Tsang, J.A. Kash and D.P. Vallett, "Time-resolved optical characterization of electrical activity in integrated circuits", in Proceedings of the IEEE, Vol. 88,No. 09, pp. 1440-1459, 2000.

[10] K. Yang, P.B. Kateshi and J.F. Whiteker, "Electric field mapping system using an optical-fiber-based electrooptic probe", Microwave and wireless Components Letters, IEEE, pp. 164-166, vol. 11, Issue 4, Apr. 2001.

[11] B. Pannetier, P. Lemaitre-Auger, S. Tedjini, E. Dogheche, D. Remiens, "External Optical Probe for Novel Online Testing Method of Digital Circuits", URSI-GA 2002, august 2002, Maastricht, Netherlands.

[12] Aktouf .Chouki, Pannetier Benoît, Pierre Lemaître-Auger \& Smail Tedjini "On-line Testing of embedded Systems Using Optical Probes: System Modeling and Probing technology", Proceedings of the Eighth IEEE International On-Line Testing Workshop (IOLTW'02), 2002.

[13] Abdelhakim Latoui \& Farid Djahli, "An Optical BILBO for On-Line Testing of Embedded Systems", IEEE Design \& Test, Vo. 30, No. 3, June. 2013, pp. 34-48.

[14] Al-assad.H, Murray.B and Hayes.J "Online BIST for Embedded systems", IEEE Design \& Test of Computers, pp.17-24, 1998.

[15] Al-Asaad, H., "Efficient Techniques for Reducing Error Latency in Online Periodic Built-in Self-Test", IEEE Instrumentation \& Measurement Magazine, pp. 28-32, 2010.

[16] B. Koenemann, J. Mucha and G. Zwiehoff, "Built-In Test for Complex Digital Integrated Circuits," IEEE J. Solid State Circuits, Vol. 15, No. 3, pp. 315-318, June 1980.

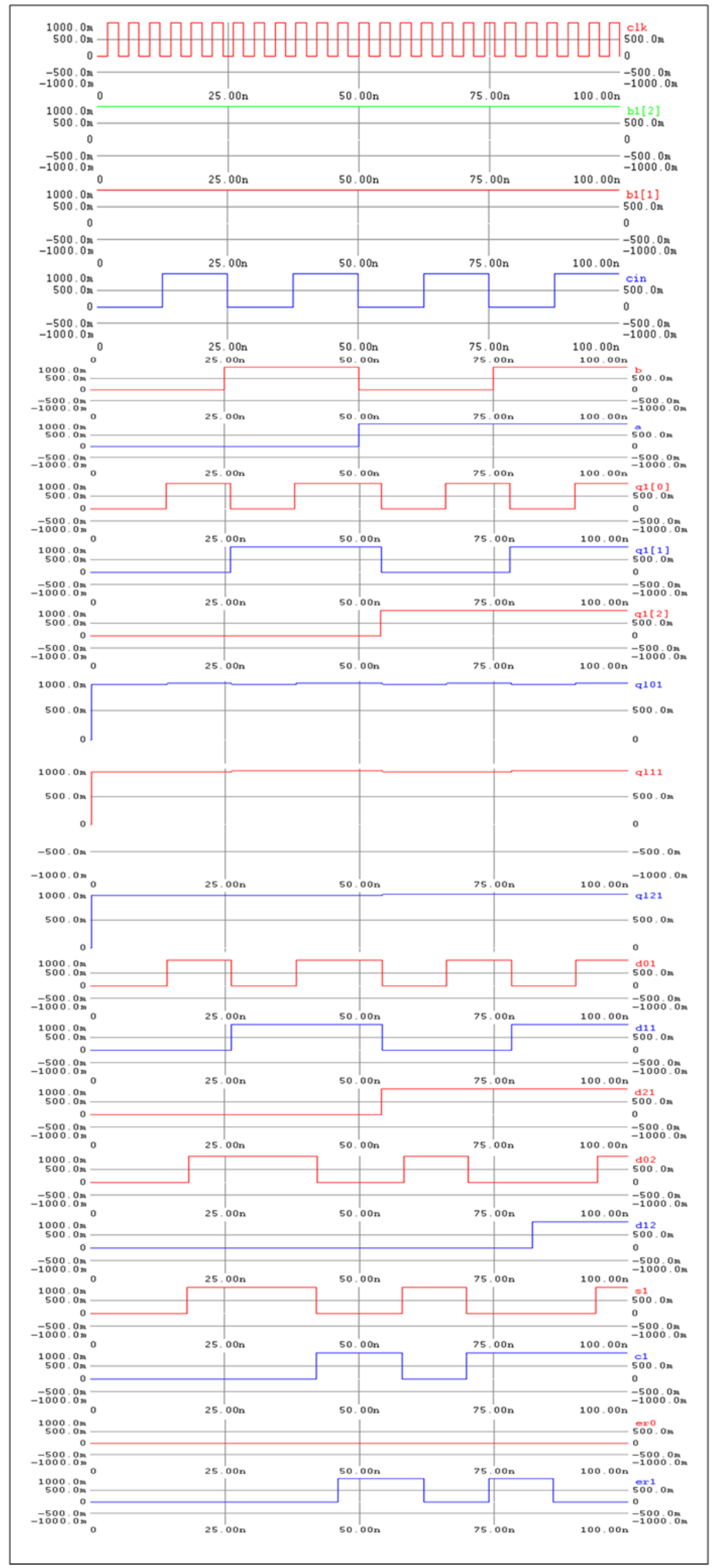

Figure 6. Simulation of the proposed model. 